zu lesen wäre gewesen, wenn Long und Kollegen Lösungen für die Senkung des Risikos des Performancebias in nichtpharmakologischen Studien diskutiert hätten, in denen Patienten teilweise und Therapeuten generell nicht verblindet werden können. Eine detaillierte Betrachtung dieses Themas findet sich bei Haller et al. [5]. Schließen möchte ich mit dem Ausblick und der Hoffnung, dass sich Forscher komplementärer Verfahren für Fragen der (spezifischen) Effektivität vermehrt der Sprache und wissenschaftlichen Richtlinien von Cochrane und STRICTA bemächtigen und kreative Lösungen zur Verbesserung der Studienqualität finden. Für andere wissenschaftliche und praktische Fragestellungen bedarf es anderer Ansätze für Studiendesigns, die nicht strikt zwischen spezifischen und unspezifischen Therapieeffekten zu trennen versuchen und die nachgewiesenen, positiven Wirkungen des sogenannten Placeboeffekts eruieren und aktiv einbeziehen.

Literatur

1. MacPherson H, Altman DG, Hammerschlag R, Youping L, Taixiang W, White A et al (2010) Revised standards for reporting interventions in clinical trials of acupuncture (STRICTA): extending the CONSORT statement. PLOS Med 7(6):e1000261. https://doi.org/10.1371/journal.pmed.1000261

2. Higgins JPT, Thomas J, Chandler J, Cumpston M, Li T, Page MJ, Welch VA (Hrsg) (2019) Cochrane Handbook for Systematic Reviews of Interventions version 6.0 (updated July 2019). www.training.cochrane.org/handbook. Zugegriffen:3. Okt. 2020
3. Hou T, Zheng Q, Feng X, Wang L, Liu Y, Li Y (2020) Methodology and reporting quality evaluation of acupuncture for mild cognitive impairment: an overview of systematic reviews. Evid Based Complement Alternat Med. https:// doi.org/10.1155/2020/7908067

4. Luo S, Long Y, Xiao W et al (2020) Risk of bias assessments and reporting quality of systematic reviews and randomized controlled trials examining acupuncture for depression: An overview and meta-epidemiology study [published correction appears in J Evid Based Med. 2020 Aug;13(3):249. J Evid Based Med 13(1):25-33. https://doi.org/10.1111/jebm.12372

5. Haller H, Lauche R, Sundberg T, Dobos G, Cramer H (2019) Craniosacral therapy for chronic pain: a systematic review and meta-analysis of randomized controlled trials. BMC Musculoskelet Disord 21(1):1. https://doi.org/10.1186/s12891-019$3017-y$

\section{Korrespondenzadresse}

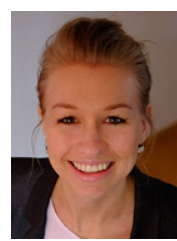

Dr. rer. medic. Heidemarie Haller

Klinik für Naturheilkunde und Integrative Medizin, Evang. Kliniken Essen-Mitte,

Medizinische Fakultät, Universität Duisburg-Essen Am Deimelsberg 34 a, 45276 Essen, Deutschland h.haller@kem-med.com

Interessenkonflikt. H. Haller gibt an, dass kein Interessenkonflikt besteht.

\title{
Erratum zu: Eine Analyse der methodischen Qualität von Akupunkturstudien der letzten 50 Jahre
}

\section{Erratum zu:}

Dtsch Z Akupunkt 2020

https://doi.org/10.1007/s42212-020-00319-8

In der Online-Version des ursprünglich publizierten Artikels wurde der englische Beitragstitel falsch ausgespielt. Der Fehler wurde korrigiert, wir bitten um Beachtung.

Die Redaktion

\section{Korrespondenzadresse}

\section{Dr. rer. medic. Heidemarie Haller} Klinik für Naturheilkunde und Integrative Medizin, Evang. Kliniken EssenMitte, Medizinische Fakultät, Universität Duisburg-Essen Am Deimelsberg 34 a, 45276 Essen, Deutschland h.haller@kem-med.com

Die Online-Version des Originalartikels ist unter https://doi.org/10.1007/ s42212-020-00319-8 zu finden.

Deutsche Zeitschrift für Akupunktur 2021 • 64 (1): 28 https://doi.org/10.1007/s42212-020-00331-y Online publiziert: 17. Dezember 2020

๑) Springer Medizin Verlag GmbH, ein Teil von Springer Nature 2020 